Cahiers $d u$ MONDE RUSSE

\section{Cahiers du monde russe}

Russie - Empire russe - Union soviétique et États indépendants

$43 / 4 \mid 2002$

Intellectuels et intelligentsia

\title{
Andrea Graziosi, Guerra e rivoluzione in Europa, 1905-1956
}

\section{Alessandro Stanziani}

\section{(2penEdition}

Journals

Édition électronique

URL : https://journals.openedition.org/monderusse/4064

DOI : 10.4000/monderusse.4064

ISSN : $1777-5388$

\section{Éditeur}

Éditions de l'EHESS

\section{Édition imprimée}

Date de publication : 30 décembre 2002

Pagination : 815-819

ISBN : 2-7132-1796-2

ISSN : $1252-6576$

Référence électronique

Alessandro Stanziani, «Andrea Graziosi, Guerra e rivoluzione in Europa, 1905-1956 », Cahiers du monde russe [En ligne], 43/4 | 2002, mis en ligne le 18 juin 2009, consulté le 04 septembre 2022. URL : http://journals.openedition.org/monderusse/4064; DOI : https://doi.org/10.4000/monderusse.4064

Ce document a été généré automatiquement le 4 septembre 2022

Tous droits réservés 


\title{
Andrea Graziosi, Guerra e rivoluzione in Europa, 1905-1956
}

\author{
Alessandro Stanziani
}

\section{RÉFÉRENCE}

Andrea GRAZIOSI, Guerra e rivoluzione in Europa, 1905-1956. Bologne, Il Mulino, 2001,327 p.

1 Voici un ouvrage riche, dense, qui ne manquera pas de susciter la discussion. Il traite en effet de la «Grande Guerre européenne », terme par lequel Andrea Graziosi essaie de rendre compte des phénomènes multiples ayant secoué l'Europe pendant la première moitié du $\mathrm{xx}^{\mathrm{e}}$ siècle : deux guerres mondiales, le nazisme et le fascisme, la révolution russe et le stalinisme, l'effondrement des empires et la question nationale. Tous ces phénomènes sont reliés par un facteur commun, à savoir l'État, sa construction, ses idéologies. Tentons de comprendre de quelle manière.

2 Le découpage chronologique avancé dans le titre (1905-1956) constitue une thèse en luimême. En amont, 1905 compte moins pour la première révolution russe que l'événement qui l'a déclenchée, c'est-à-dire la défaite russe dans la guerre avec le Japon. Cette issue bouleverse tous les équilibres, tant en Russie et dans l'Empire austro-hongrois que, suite à l'éclatement de ce dernier, dans le reste de l'Europe. En aval, 1956 marque la fin du stalinisme, l'essor de la décolonisation et, de ce fait, la fin des anciens États-nations (Grande-Bretagne et France en particulier). Une fois de plus, les événements à l'Est et à l'Ouest sont étroitement liés entre eux.

Afin de démontrer cette thèse, l'auteur a conçu un ouvrage composé de trois parties principales, dont la première («Quoi expliquer et comment») fait état des principales interprétations des régimes dominants $\mathrm{au} \mathrm{xx}^{\mathrm{e}}$ siècle. La deuxième partie identifie les origines politiques, démographiques, économiques et culturelles des phénomènes du $\mathrm{xx}^{\mathrm{e}}$ siècle. La troisième, enfin, examine en détail la "grande guerre-révolution » du 
XXe siècle, à l'intérieur de laquelle trois périodes sont distinguées : 1905-1923 ; 1923-1939 ; 1939-1956.

4 Graziosi commence par rappeler comment, dès le premier après-guerre, deux interprétations s'imposent, l'une qui regarde favorablement l'essor de l'État lié à la Première Guerre mondiale et l'autre qui le critique. Les courants socialistes et les dirigeants allemands se rattachent à la première, tandis que Mises constitue le champion de la seconde. Dans ce cadre, l'historique de la notion de totalitarisme (à commencer par l'Italie fasciste) représente une des parties fortes de l'ouvrage ${ }^{1}$. Graziosi montre l'incapacité de ce concept à expliquer les réalités non seulement italienne et allemande mais même soviétique. Il offre là une synthèse remarquable de l'évolution de ce dernier pays en prenant en considération la personnalité de Stalin, la dynamique politique, l'évolution sociale et économique, pour en arriver à récuser la pertinence du totalitarisme en tant que catégorie explicative. En s'appuyant d'une part sur Halévy et d'autre part sur Moshe Lewin, l'auteur lui préfère le terme de tyrannie.

5 La deuxième partie de l'ouvrage repère au $\mathrm{XIX}^{\mathrm{e}}$ siècle les origines des guerres et des révolutions successives. Compte tenu du rôle attribué à la construction étatique, il n'est pas surprenant que l'histoire européenne soit centrée davantage sur la France et ignore l'Angleterre. Ce choix apparaît néanmoins d'autant plus surprenant dans une histoire qui se veut européenne. Même si l'auteur est conscient des limites de cette exclusion, il n'avance aucun argument vraiment convaincant pour la justifier. Encore faut-il accepter le rôle attribué à l'État dans l'histoire française (l'auteur parle même de nationalisations (p.100) pour le $\mathrm{xIX}^{\mathrm{e}}$ siècle), rôle qui est loin d'être entériné par l'historiographie, du moins dans cette version simplifiée.

6 En réalité, dans l'architecture de l'ouvrage, l'étatisme français s'ajoute à celui des empires d'Europe centrale et orientale afin de rendre compte de la persistance d'éléments d'Ancien Régime dans l'Europe du XIx siècle (paysans et élites nobles essentiellement). Pour sa part, le succès de l'industrialisation allemande, dirigée par l'État, permet d'expliquer la diffusion de ce modèle dans d'autres pays émergents de l'époque. Ce schéma aurait finalement aveuglé les socialistes au point qu'ils auraient fini par associer industrialisation (étatique et à n'importe quel prix) et modernisation.

7 À plusieurs reprises, l'auteur oppose ce rôle de l'État, source d'oppression et de distorsion, voire de dégénérescence, à celui du libre marché. Nous trouvons là une approche néo-libérale très en vogue aujourd'hui. Il est néanmoins symptomatique que l'adhésion à cette thèse aille jusqu'à ignorer le rôle politique joué par les groupes entrepreneuriaux, tant en Allemagne qu'en Italie, dans la montée du nazisme et du fascisme. De manière générale, l'opposition entre l'État et le marché oublie l'attitude toujours ambiguë à cet égard (dès l'époque moderne et encore plus après la Révolution française) des milieux d'affaires. Je renvoie au livre magnifique de Jean-Pierre Hirsch pour rendre compte de cet aspect (Les deux rêves du commerce, Paris, EHESS, 1991).

Plus convaincante est la partie dans laquelle Graziosi arrive à faire jouer à la fois les variables démographiques, sociales et politiques, pour rendre compte de l'histoire européenne $\mathrm{du} \mathrm{XIX}^{\mathrm{e}}$ siècle, et en particulier de la convergence entre nationalisme et socialisme. Dans ce contexte, l'analyse de l'Europe orientale (Ukraine et Pologne en particulier), où le rôle des composantes ethniques, religieuses, culturelles et politiques est correctement détaillé, constitue une des contributions les plus importantes de l'ouvrage. 
9 Le cadre dressé pour la Turquie et pour les Balkans est également riche et, surtout, la réinterprétation de l'histoire européenne à partir d'une perspective orientale est stimulante, tant pour les thèses avancées que pour la méthode adoptée. Il s'agit sans doute de l'un des meilleurs chapitres du livre, qui reflète la longue expérience accumulée par Graziosi sur ce terrain. La seule question qui pourrait être soulevée ici concerne l'usage du terme d'Europe orientale. L'auteur a recours à la définition de Mises, qui se révèle par la suite un puissant outil explicatif. Mais en même temps, cela aurait peut-être valu la peine de justifier ce choix et de rappeler brièvement de quelle manière il se place dans l'historique complexe du terme à des moments différents, par des disciplines différentes (géographie, histoire, sciences politiques), dans des réalités également différentes (par exemple, la définition de l'Europe orientale donnée en Hongrie n'est pas la même qu'en Roumanie).

10 La troisième partie est celle qui donne son titre à l'ouvrage. Dans sa construction globale, comme dans ses thèses multiples, elle offre une synthèse courageuse, efficace et novatrice du $\mathrm{xx}^{\mathrm{e}}$ siècle. Par exemple, la révolution russe de 1905 est rapprochée du printemps des peuples de 1848, c'est-à-dire qu'elle est analysée dans un rapport entre empire et nationalités (p.162). L'auteur remarque avec pertinence qu'en Europe occidentale, à la différence de l'Europe orientale, la Première Guerre mondiale n'a jamais remis en cause l'existence des États-nations; il n'empêche que ce conflit signe l'arrêt de mort définitif de l'Ancien Régime. Graziosi inclut dans un même processus l'effondrement des empires tsariste, austro-hongrois et allemand.

11 L'entre-deux-guerres répond aux convulsions déclenchées par le conflit; à ce sujet, l'auteur parvient à étudier ensemble des éléments trop souvent analysés séparément, à savoir les tendances sociales et économiques de longue durée et les personnalités des despotes. C'est dans cette perspective qu'il assure une analyse stimulante du lien entre nationalisme et socialisme, tant dans les idéologies que dans les pratiques politiques. Audelà des différences, Graziosi souligne les analogies entre l'Allemagne nazie et l'URSS de Stalin : des phénomènes communs à l'origine, la force du nationalisme, le rôle de l'État. C'est à partir de ce constat qu'il peut critiquer l'idée de guerre civile européenne.

Cette troisième partie - 1939-1956 - constitue donc une fresque riche et complexe dans laquelle les événements tragiques (guerre mondiale, déplacements forcés, etc.) sont expliqués et intégrés, à l'Est, dans l'héritage des questions nationales et, à l'Ouest, dans la fin des sociétés paysannes et dans la décolonisation.

13 Le comparatisme constitue un élément important de cet ouvrage. Il est exercé de manière intelligente, c'est-à-dire en ayant moins recours à un modèle théorique qu'à l'analyse historique proprement dite. Le choix des pays est significatif : à la différence de la quasi-totalité des histoires comparées du $\mathrm{xx}^{\mathrm{e}}$ siècle, celle de Graziosi accorde une place significative à des pays comme l'Ukraine, trop souvent incluse dans l'histoire soviétique. La perspective est à la fois plus stimulante et plus pertinente pour rendre compte des événements qui ont bouleversé l'Europe jusqu'à nos jours.

14 L'auteur utilise l'expérience soviétique comme pierre d'achoppement pour analyser d'autres réalités. La question qui se pose dès lors est celle de la valeur heuristique du cas soviétique. Comme, le plus souvent, les historiens spécialistes de l'URSS se cantonnent derrière une "spécificité " de leur aire culturelle et évitent toute comparaison, on ne peut qu'accueillir favorablement la tentative de Graziosi. Celui-ci critique en particulier le fait que les pays étudiés aient à tort été rapprochés sous l'angle du nationalisme, alors que le vrai terme de la comparaison est la construction étatique qui, seule, permet de 
rendre compte de la dégénérescence du nationalisme, du socialisme et du libéralisme. Cependant, ce résultat ne fait que déplacer les termes du problème, car l'auteur identifie l'État tantôt à la bureaucratie, tantôt à l'appareil administratif, et il avoue que ces distinctions mériteraient d'être développées ultérieurement. Cette conclusion laisse le lecteur sur sa faim, étant donné le rôle absolument crucial accordé à l'« État ». En réalité, ce problème ne concerne pas que l'ouvrage de Graziosi ; il est plus général. Comme l'État, la bureaucratie, l'administration rentrent dans la quasi-totalité des analyses historiographiques, politiques, sociologiques et anthropologiques $\mathrm{du} \mathrm{xx}^{\mathrm{e}}$ siècle, le moment semble venu de mettre de l'ordre dans ces catégories.

La conclusion confirme les capacités de synthèse et d'analyse de l'auteur. Les transformations en Europe occidentale et orientale ainsi que dans le "Tiers-Monde " sont liées. Le dernier acte de la Grande Guerre européenne aura ainsi des résultats tout à fait opposés au premier: les tyrannies s'effondrent, les empires coloniaux aussi et la construction européenne devient une réalité. C'est-à-dire que la guerre n'est pas forcément un facteur de déstabilisation, mais que des « guerres justes » sont possibles.

Cette conclusion, qui prête à débat, reflète la conception de l'auteur pour lequel l'histoire est jugement: s'en "abstenir serait non seulement impossible, mais même scientifiquement erroné » (p. 22). C'est sur cette base que le $\mathrm{xx}^{\mathrm{e}}$ siècle devient une lutte du bien (la liberté) contre le mal (stalinisme, nazisme, maoïsme, etc.). D'une manière générale, les jugements de valeur sont extrêmement nombreux dans ce livre, ils en conditionnent l'architecture et les thèses principales. Cette querelle ancienne (l'histoire juge ou pose des interrogations), somme toute propre au métier d'historien, a acquis une virulence particulière à l'époque contemporaine. La réponse donnée conditionne l'approche des sources. C'est pourquoi l'auteur déclare s'inspirer de l'idéal de Ranke, qu'il traduit par « la nécessité pour tout historien qui souhaite être tel de reconstruire, dans les limites du possible, le passé tel qu'il a réellement été » (p. 28). Graziosi oppose cette démarche au «relativisme radical » qui «a provoqué de si nombreux dommages au cours des dernières années » (p. 28). La question est complexe dans la mesure où, si le relativisme risque de cautionner le négationnisme (mais il faudrait souligner avec force l'écart entre les deux démarches), en contrepartie le retour au positivisme des sources risque de faire oublier que les archives elles-mêmes sont bâties, classées, et ensuite sélectionnées et lues dans un cadre historique, politique et épistémologique donné. Cet élément semble d'autant plus indispensable au moment où, comme dans cet ouvrage, le rôle de l'État devient la variable explicative fondamentale du processus historique. L'auteur aurait peut-être mieux fait d'éviter de rentrer dans ce débat méthodologique, étant donné la nature de son ouvrage ( « celui-ci n'est pas, ni ne veut être, un livre d'histoire ", mais plutôt un ensemble de réflexions sur l'histoire européenne du $\mathrm{xx}^{\mathrm{e}}$ siècle, p. 9). En même temps, nous ne pouvons que lui être reconnaissants d'avoir mis à l'ordre du jourune question méthodologique qui, après l'école des Annales et le déconstructivisme, et surtout à la suite de l'ouverture des archives ex-soviétiques, mériterait un regain d'attention plus important. Ces réflexions méthodologiques confirment l'intérêt extrême d'un ouvrage dont nous ne pouvons que souhaiter la traduction en d'autres langues, à commencer par le français. 


\section{NOTES}

1. Même si l'auteur aurait mieux fait d'éviter des chutes de ton comme celle de la page 65 où il s'en prend à la gauche qui, tardivement, aurait eu recours à la notion de totalitarisme pour « exorciser son propre passé ». 